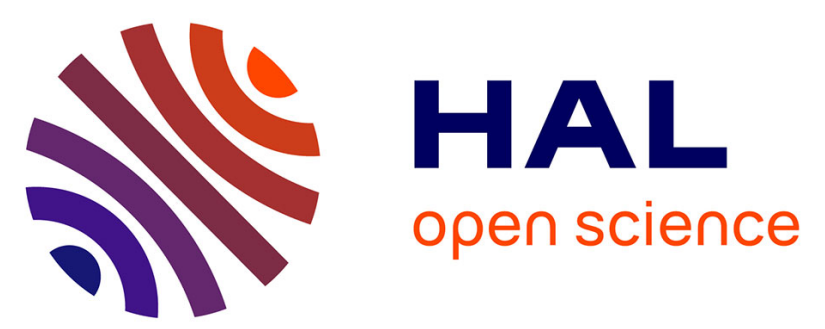

\title{
Exploring the possibility of predicting CALB activity in liquid organic medium, with the aid of intrinsic kinetic parameters and intrinsic solvent effect data obtained in solid/gaz reactor.
}

Marianne Graber, Valérie Léonard, Zsuzsanna Marton, Claire Cusatis, Sylvain

Lamare

\section{To cite this version:}

Marianne Graber, Valérie Léonard, Zsuzsanna Marton, Claire Cusatis, Sylvain Lamare. Exploring the possibility of predicting CALB activity in liquid organic medium, with the aid of intrinsic kinetic parameters and intrinsic solvent effect data obtained in solid/gaz reactor.. Journal of Molecular Catalysis B: Enzymatic, 2007, 52-53, pp.121-127 June 2008. hal-00329741

\section{HAL Id: hal-00329741 \\ https://hal.science/hal-00329741}

Submitted on 13 Oct 2008

HAL is a multi-disciplinary open access archive for the deposit and dissemination of scientific research documents, whether they are published or not. The documents may come from teaching and research institutions in France or abroad, or from public or private research centers.
L'archive ouverte pluridisciplinaire HAL, est destinée au dépôt et à la diffusion de documents scientifiques de niveau recherche, publiés ou non, émanant des établissements d'enseignement et de recherche français ou étrangers, des laboratoires publics ou privés. 
1 Exploring the possibility of predicting CALB activity in liquid

2 organic medium, with the aid of intrinsic kinetic parameters and

3 intrinsic solvent effect data obtained in solid/gaz reactor.

4

5

6

7

Marianne GRABER*, Valérie LEONARD, Zsuzsanna MARTON, Claire CUSATIS and Sylvain LAMARE

Université de la Rochelle, Laboratoire de Biotechnologies et Chimie Bio-organique, CNRS

FRE 2766, Pôle Sciences et Technologies, Bâtiment Marie Curie, Avenue Michel Crépeau, 17042 La Rochelle, Cedex 1, France

*Corresponding author : Dr. Marianne GRABER, tel: 33546458630 , fax: 33546458265 , E-mail: mgraber@univ-lr.fr 


\section{Abstract}

In organic solvents the activity of enzyme is often order of magnitude lower than in water solutions and varies significantly with the solvent used. Many attempts to quantify and model the decrease of enzyme catalytic efficiency in solvents have been performed, but still there is no general method for predicting enzyme activity in a particular solvent in absolute terms.

In this paper, a new method is proposed to predict initial reaction rates in different anhydrous monophasic liquid organic media, in the case of CALB-catalyzed alcoholysis

11 reaction. The experimental tool used for this method is a solid/gas bioreactor, which allows to obtain enzyme intrinsic kinetic parameters and intrinsic effect of organic solvents on enzyme activity. It is shown that, once corrections for solvation of substrates have been performed, the effect of solvent molecules consists mainly on binding to enzyme active site, leading to competitive inhibition of solvent towards the first substrate ester. The intrinsic parameters obtained in solid/gas reactor are used to predict activity of different batches of CALB, in organic media of different polarities, based on a single rate measurement in hexane. The result is a right prediction of solvent order with regard to CALB activity, a proper approximation of initial rate for weakly inhibiting solvents and overestimation for solvents showing a marked inhibitor character.

23 Keywords: Lipase B from Candida antarctica; alcoholysis; organic solvent; solid/gas biocatalysis; competitive inhibition. 


\section{Introduction}

Enzyme catalysis in organic anhydrous solvents has been a topic of research which has been extensively explored over the last decades [1-4]. For a large number of enzymes and especially for lipases, these solvents possess great advantages: increased solubility of hydrophobic substrates, increased thermostability of the enzyme and shift of the thermodynamic equilibrium in favor of synthesis over hydrolysis.

However in organic solvents the activity of enzyme is often order of magnitude lower than in water solutions and varies significantly with the solvent used. Many studies have been performed in organic liquid media to explain this loss of activity, leading to well established different causes such as unfavorable energetics of desolvation of substrates and active site [2], transition state destabilization, loss of conformational mobility of the enzyme [5] and competition of solvent molecules with substrate for binding [6]. This last effect has often been considered as a major cause of reduced activity of enzymes in organic liquid media [6-8]. Attempts to quantify and predict the decrease of enzyme catalytic efficiency in solvents include, for example, correction of substrates and kinetic parameters for solvation [7], prediction of the enzymatic activation energy depending on solvent polarity [9], or addition of an initial equilibrium step in the catalytic pathway [10]. In these methods, kinetic and thermodynamic parameters are determined in a particular solvent (in general a polar solvent) for an enzyme catalyzed reaction and the differences likely to be obtained for these parameters between another solvent and this particular solvent, are predicted from dissimilarities of physico-chemical properties between the two solvents, like saturation solubility or partioning between the two solvents for a substrate. Consequently these methods do not permit to predict enzyme activity in a particular solvent in absolute terms. 
In the present paper, a way of determining the intrinsic effect of solvent in the case of

an immobilized lipase-catalyzed alcoholysis reaction is proposed. The experimental tool used to determine this parameter is a solid/gas bioreactor. In this system, the immobilized enzyme forms a packed bed, percolated by nitrogen as carrier gas, which simultaneously carries gaseous substrate to the enzyme and removes reaction products. This type of reactor allows a precise control of the enzyme microenvironment and permits determination of the sole role of the addition of an organic compound [11-13]. It was previously shown that kinetic parameters obtained by using this kind of reactor, can be considered as "intrinsic" kinetic parameters, as enzyme activities are measured in absence of both solvent and diffusional limitations $[12,13]$.

In the following work, we tested the possibility of predicting in absolute terms, the enzymatic reaction rates, in a totally anhydrous monophasic liquid organic medium, thanks to the kinetic intrinsic parameters and the intrinsic solvent effect data obtained in solid/gas reactor.

3

\section{Theoretical Analysis}

In this study, the effect of monophasic organic anhydrous solvents on enzyme catalysis is considered to arise from two different causes: (1) changes in solubility and desolvation of free substrates and products; (2) changes of solvation of enzyme species (free or as enzyme-substrate complex) and in particular binding of solvent molecules on enzyme active site. The possibility of either quantifying or canceling these effects thanks to the use of the solid/gas enzymatic system is explained in the following part.

The ability of solvents to change enzyme conformation and/or flexibility $[5,14]$ is not considered here. Indeed, in the case of the enzyme chosen for testing our model, i.e. Candida antarctica lipase B (CALB), no significant conformational change was displayed when studying lipase structure in different solvents (2-pentanone, 2-methyl-2-pentanol and 2- 
1 methyl pentane) by molecular modeling [15]. It is also assumed that solvents have no effect

2 on the catalytic mechanism of the enzyme. This hypothesis was supported by kinetic results

3 obtained in different kind of non conventional media for CALB, which could all be modelized

4 by the same mechanistic model $[12,16]$.

\subsection{Effect of solvents on free substrates and products solubility and desolvation}

One of the main effects of solvents on enzyme catalysis arises from solvent interaction with substrates and products, by solvation and desolvation phenomena, which modifies substrate and products availability for the enzyme $[17,18]$. Different methods have been proposed for correcting for these effects [8, 19-22]. One of them consist in expressing the quantities of substrate and the kinetic constant in terms of thermodynamic activities instead of concentrations $[21,22]$. To perform such corrections, activity coefficients ( $\gamma$-values) of substrates are needed; they are either found from values in data bases, determined experimentally or estimated by using the UNIFAC group contribution method [23]. Nevertheless, authors frequently call UNIFAC predictions into question, as sources of inaccurate $\gamma$-values, being the cause of differences in enzyme performance observed in various solvents $[6,7,21]$. Deviations in the UNIFAC calculations up to a factor of two have been reported [21]. Moreover, in most of the experiments, $\gamma$-values are determined for one set of substrates and solvent quantities and considered to be constant in the range of concentrations used. These approximations lead to potentially cumulative errors on kinetic constant determination.

Consequently it appears that correction for solvation of substrates is far from being straightforward $[19,22]$. Therefore determination of intrinsic kinetic parameter of enzymes, i.e. independent of solvent used, is not possible.

To circumvent this problem, the use of enzymes at the solid/gas interface appears concurrent to liquid processes and presents some very interesting features. Indeed, contrary to the 
1 classical solid/organic liquid system, the solid/gas process offers the possibility to perfectly

2 control and adjust thermodynamic activities of both reaction species and non-reactant 3 components. A complete explanation of the design of solid/gas reactor and of the theoretical background for thermodynamic activities control has been presented previously [11]. In this system, the thermodynamic activity of each compound is determined by calculating the ratio of the partial pressure of the compound in the gas to its saturation vapor pressure at the working temperature, assuming that the poorly charged inert inlet gas can be considered as an ideal gas. The partial pressure is obtained from molar composition of the inlet gas and the total pressure. As molar fraction of the different species in the inlet gas can be chosen independently, thermodynamic activities of these species can be fixed independently very easily. This constitutes a significant advantage over solid-liquid system, in which reaction species and solvent molar fractions are linked together and for which totally inert liquid solvents do not exist.

\subsection{Effect of solvents on the energy of desolvation of the active site, on the activation energy} of enzyme catalysis and on the extent of solvation of the enzyme species (free or as enzymesubstrate complex).

The ability of solvent molecules to bind in the active site of free enzymes and to the covalent reaction intermediates, has been studied by many authors, in order to explain why enzymatic activity is often much reduced in organic solvents compared with water.

As an example, Schmitke et al. [24] have compared X-ray crystal structures of an acylenzyme intermediate of subtilisin Carlsberg formed in anhydrous acetonitrile and water. It appears that the structures of this covalent intermediate (trans-cinnamyl-subtilisin) did not change upon formation in either acetonitrile or water, and that the free enzyme active site structure remained unchanged when using water or acetonitrile. Therefore the solvent would not change the activity of subtilisin by causing a conformational change in the active site or 
1 by affecting the mode of binding of the substrate. However the locations of bound solvent

2 molecules in the active site of the acyl- and free enzyme forms in acetonitrile and in water are 3 distinct, leading to different energies required to displace these solvent molecules to allow substrate binding and catalysis. Such differences may contribute to the observed variations in enzymatic activities in water and acetonitrile.

The effect of solvents on the activation energy of enzyme catalyzed reaction has been noticed by several authors. For example, it was found that polar solvents stabilize subtilisin's transition state which is polar in nature, as it is charged. This stabilization lowers the intrinsic activation energy of subtilisin-catalyzed transesterification reactions, leading to higher activities in such solvents [9]

Finally, as mentioned by Van Tol et al.[7], there are many possible causes for the effects of the solvents on the catalytic performance of the enzymes, remaining after correction for substrate-solvent interactions and the amount of participating enzyme : $1^{\circ}$ ) dependence of the energy of desolvation of the active site of the nature of the solvent, $2^{\circ}$ ) possible solvation of bound substrate, $3^{\circ}$ ) activity coefficients of the different enzyme species in the catalytic cycle not constant, $4^{\circ}$ ) effect of solvents on the local polarity in the active site and $5^{\circ}$ ) competition of solvents with substrates for binding

All these possible effects render the interpretation of kinetic and thermodynamic measurements in liquid media quite hazardous, because of the presence of such large quantities of solvent molecules.

In the solid/gas enzymatic systems the addition of solvent is avoided and the microenvironment of the enzyme is solely composed of substrates and products molecules, provided that influence of other proteins and support material for immobilization can be neglected. In the present study, a low enzyme to support ratio was used, allowing to discard interactions between proteins and it was checked that the support was sufficiently inert towards adsorption to avoid interference with kinetic results. Therefore solid/gas system 
1 renders possible to measure enzyme activity in the absence of non reactant component. The

2 only additional component present in solid/gas enzymatic systems is the carrier gas, which

3 carries gaseous substrates to the enzyme and removes gaseous products and residual

4 substrates. In the present study, gaseous nitrogen was used as carrier gas and numerous

5 experiments showed that it had no effect on enzyme catalyzed reaction rate and could

6 therefore be considered as a totally inert component towards enzyme kinetics. reactant component, at a fixed thermodynamic activity, in addition to substrates, whose quantity is independently controlled. This allows to assess the effect of the addition of this component alone and to quantify its "intrinsic" effect on enzyme activity.

11

12

13

Besides it is possible to add in the gaseous feed of the solid/gas reactor, an extra non-

(1)

\section{Experimental}

\subsection{Enzyme and chemicals}

CALB used in part 4.1 and 4.2 was Chirazyme L2 (Type B lipase from Candida antarctica lyophilized), Roche Industrie (Penzberg, Germany), immobilized by adsorption onto Chromosorb P AW DMCS, mesh 60-80 (Acid Washed DiMethylChloroSilane) (Varian, France) as described in a previous work [16] and then stored at $4^{\circ} \mathrm{C}$ over silica gel. $1 \mathrm{mg}$ of adsorbed enzyme preparation corresponded to $3.32 \mu \mathrm{g}$ of lyophilized lipase.

Batch B of immobilized CALB used in part 4.3 consisted of Lipozyme CALB L (Novozymes) dialyzed at $4^{\circ} \mathrm{C}$ during $48 \mathrm{~h}$ with $20 \mathrm{mM} \mathrm{pH} 7.5$ phosphate buffer, then three times concentrated under vacuum and immobilized by adsorption on Chromosorb P AW DMCS. 
1 All chemicals were of the highest purity (99\% minimum), dried prior to use and stored under

2 argon atmosphere and over molecular sieves.

3

\subsection{Experimental setup for solid-gas catalysis and chromatographic assays}

The bioreactor used in this study has already been described in a previous publication $[11]$

Intrinsic kinetic parameters and kinetic parameters in presence of 2-methyl-2-butanol were determined in solid/gas reactor at $70^{\circ} \mathrm{C}$ as described previously $[12,16]$. Intrinsic inhibition constants of solvents were obtained as previously explained $[12,15]$.

The vapor phase leaving the bioreactor was sampled, automatically injected and analyzed by gas chromatography as previously described $[12,15,16]$.

\subsection{Experimental setup for reactions run in organic phase and chromatographic assays}

Kinetic study in 2-methyl-2-butanol (part 4.1) was performed as previously described [16].

Reactions run in liquid water, 2-methyl-2-butanol, 2-pentanone, 3-pentanone, 3-hydroxy-3methylpentane (part 4.3) were carried out in sealed flasks using $62.5 \mathrm{mg}$ of adsorbed enzyme and $5 \mathrm{ml}$ of a solution containing methyl propanoate, propanol and 2-methyl-2-butanol at specified thermodynamic activities. The mixture was incubated at $70^{\circ} \mathrm{C}$ under magnetic stirring. Samples were taken at intervals and analyzed by gas chromatography for quantitative determination of products and residual substrates, as previously described [16], except that the initial column temperature was $35^{\circ} \mathrm{C}$, then kept at $35^{\circ} \mathrm{C}$ during $7.5 \mathrm{~min}$ and finally programmed to increase at $30^{\circ} \mathrm{C} / \mathrm{min}$ to $95^{\circ} \mathrm{C}$. For reaction run in hexane, four samples of reaction medium were prepared in sealed flasks and incubated with the enzyme during different times. 


\section{Results and Discussion}

2

\subsection{Determination of the intrinsic effect of a particular solvent (2-methyl-2-butanol) for}

\section{CALB-catalyzed alcoholysis reaction}

In the following part, kinetic data obtained in previous papers were used to calculate intrinsic effect of different solvents on CALB activity. The reaction model studied is alcoholysis of methyl propanoate and $n$-propanol catalyzed by CALB. Complete kinetic studies were performed in solid/gas reactor, both in absence of any added component except the carrier gas nitrogen [16] and in presence of a fixed added 2-methyl-2-butanol quantity in the carrier gas [12]. The same reaction was also studied in liquid 2-methyl-2-butanol [16]. Alcoholysis reactions of gaseous substrates were found to obey the same kinetics mechanism than in organic liquid medium: Ping Pong $\mathrm{Bi} \mathrm{Bi}$ mechanism with dead-end inhibition by the alcohol $[12,16]$

In all these studies, substrate quantities were expressed in terms of thermodynamic activities, in order to better quantify the availability of substrates to the enzyme and to make results obtained in gaseous and liquid medium comparable. This led to kinetic parameters also expressed in thermodynamic activities. For reactions in liquid organic medium, thermodynamic activities were calculated by using the UNIFAC group contribution method. As previously explained, the solid/gas reactors allow to measure enzyme activities in absence of both solvent and diffusional limitations. Therefore, kinetic parameters obtained in the gas reactor can be considered as "intrinsic parameters" of the enzyme.

Intrinsic propanol inhibition constant $\left(K_{I_{P}}^{\circ}\right)$ and enzyme-substrate dissociation constants ( $K_{M P}^{\circ}$ for methyl propanoate and $K_{P}^{\circ}$ for propanol) are given in Table 1 , as the corresponding kinetic parameters obtained in the gaseous medium in presence of a fixed thermodynamic activity of 2-methyl-2-butanol (a $\mathrm{a}_{2 \mathrm{M} 2 \mathrm{~B}}$ ) equal to 0.3 (noted $K_{I_{P}}^{2_{2} 2 B}$, 
$1 K_{M P}^{\circ 2 M 2 B}$ and $K_{P}^{\circ 2 M 2 B}$ ), and the ones obtained in liquid medium with 2-methyl-2-butanol as

2 solvent, $\left(K_{I_{P}}, K_{M P}\right.$ and $\left.K_{P}\right)$. It appears that $K_{I_{P}}$ and $K_{M P}$ are respectively, 8.4 and 13.8

3 times higher than intrinsic parameters obtained in the solid/gas reactor, whereas $K_{P}$ is

4 almost equal to $K_{P}^{\circ}$. When adding 2-methyl-2-butanol in the gas/solid reactor (with $\mathrm{a}_{2 \mathrm{M} 2 \mathrm{~B}}$ 5 equal to 0.3 ), the difference between parameters obtained in liquid and gaseous medium 6 clearly decreases, but still remains significant $\left(K_{I_{P}}\right.$ and $K_{M P}$ are respectively 3.5 and 6 7 times higher than $K_{I_{P}}^{\circ 2 M 2 B}$ and $K_{M P}^{\circ 2 M 2 B}$ ). Different assumptions explaining these results have 8 already been discussed [12], and the overall idea is that 2-methyl-2-butanol interferes with 9 the binding process between first substrate methyl propanoate and the immobilized enzyme. 10 A simple assumption is that 2-methyl-2-butanol would act as a competitive inhibitor of 11 methyl propanoate, as propanol does.

This idea of solvent as competitive inhibitor, was confirmed more recently and put 13 forward to explain the lower activity of CALB in presence of non reactant ketone or tertiary 14 alcohol in the reaction medium [15]. In this work, the clear interaction of these compounds 15 with the active site, was shown by molecular modeling, and their marked inhibitory character was confirmed by kinetic studies performed in a solid/gas reactor.

A potential effect of 2-methyl-2-butanol on $V_{\max }$ was ruled out, as $V_{\max }^{\circ}$ obtained in solid/gas reactor without any added component, was equal to $V_{\max }^{2 M 2 B}$ obtained in the same reactor at $\mathrm{a}_{2 \mathrm{M} 2 \mathrm{~B}}$ equal to 0.3 and $V_{\max }$ obtained in liquid 2-methyl-2-butanol in presence of much higher quantities of 2-methyl-2-butanol (range of $\mathrm{a}_{2 \mathrm{M} 2 \mathrm{~B}}$ between 0.33 and 0.96 ) (table1). Assuming the main effect of added 2-methyl-2-butanol on CALB-catalyzed alcoholysis is to act as competitive inhibitor of the first substrate (methyl propanoate in this case), the kinetic parameters obtained in the gas/solid reactor : intrinsic parameters on the 
1 one hand, and parameters obtained at $\mathrm{a}_{2 \mathrm{M} 2 \mathrm{~B}}$ equal to 0.3 , allow to calculate the intrinsic

2 inhibition constant of 2-methyl-2-butanol, noted $K_{I_{2 M 2 B}^{\circ}}^{\circ}$

3 By using dissociation constants between methyl propanoate and enzyme in the solid/gas

4 reactor, $K_{M P}^{\circ}$ and the same parameter obtained in gas at fixed $\mathrm{a}_{2 \mathrm{M} 2 \mathrm{~B}}$ equal to $0.3, K_{M P}^{\circ 2 M}$,

$5 \quad K_{I_{2 M 2 B}}^{\circ}$ is given by :

$6 \quad K_{I_{P}}^{\circ 2 M 2 B}=K_{I_{P}}^{\circ}\left(1+\frac{0.3}{K_{I_{2 M 2 B}}^{\circ}}\right)$, which leads to $K_{I_{2 M 2 B}}^{\circ}=0.22$

7 By the same way, using intrinsic inhibition constants of propanol $K_{I_{P}}^{\circ}$ and $K_{I_{P}}^{\circ 2 M 2 B}$, allows to

8 obtain $K_{I_{2 M 2 B}^{\circ}}^{\circ}$, as 2-methyl-2-butanol also competes with propanol, which is competitive

9 inhibitor of methyl propanoate :

$10 \quad K_{M P}^{\circ 2 M 2 B}=K_{M P}^{\circ}\left(1+\frac{0.3}{K_{I_{2 M 2 B}}^{\circ}}\right)$, which leads to $K_{I_{2 M 2 B}}^{\circ}=0.24$

11 The similarity of these two values obtained for $K_{I_{2 M 2 B}}^{\circ}$ is in accordance with our statement 12 that 2-methyl-2-butanol acts as a competitive inhibitor, and that propanol and 2-methyl-213 butanol are two exclusive inhibitors of methyl propanoate.

4.2 Prediction of initial reaction rates, for CALB-catalyzed alcoholysis, in liquid medium with 2-methyl-2-butanol as solvent, by using intrinsic parameters obtained in the solid/gas reactor

In this part we tested the ability of intrinsic kinetic parameters and intrinsic 2-methyl2-butanol effect data obtained in solid/gas reactor, for CALB-catalyzed alcoholysis reaction, to predict initial reaction rate values for the same reaction run in liquid 2-methyl-2-butanol. As previously mentioned, a complete kinetic study of CALB-catalyzed alcoholysis of methyl propanoate and propanol, was performed in liquid 2-methyl-2-butanol. Consequently a complete set of experimentally measured initial reaction rates at different substrates 
1 thermodynamic activities was available. For each of these experimental points, corresponding

2 thermodynamic activity of 2-methyl-2-butanol in the reaction medium was determined, in

3 addition to the ones of the two substrates, from molar fraction of the solvent in the medium

4 and corresponding activity coefficient coming from the UNIFAC group method. Then, each

5 measured CALB reaction rate (noted $v_{i_{\mathrm{exp}}}$ ) was compared with two different values of reaction

6 rate :

$71^{\circ}$ ) a predicted initial reaction rate (noted $v_{i_{\text {pred }}}$ ) obtained by using the classical rate equation

8 for the Ping Pong Bi Bi mechanism with dead-end inhibition by the alcohol, with intrinsic

9 kinetic parameters obtained experimentally in the solid/gas reactor, and considering that both

2-methyl-2-butanol and propanol were acting as exclusive competitive inhibitors of methyl

11 propanoate. These assumptions lead to the following equation [25] :

$$
v_{i_{\text {pred }}}=\frac{V_{\max }^{\circ} a_{P} a_{M P}}{K_{M P}^{\circ}\left(1+\frac{a_{2 M 2 B}}{K_{I_{2 M 2 B}}^{\circ}}+\frac{a_{P}}{K_{I_{P}}^{\circ}}\right) a_{P}+K_{P}^{\circ} a_{M P}+a_{P} a_{M P}} \quad \text { Equation 1 }
$$

13 where $a_{M P}$ and $a_{P}$ are respectively the thermodynamic activity of methyl propanoate and 14 propanol.

15

$2^{\circ}$ ) a calculated initial rate (noted $v_{i_{c a l c}}$ ), obtained by using the rate equation for the Ping Pong Bi Bi mechanism with dead-end inhibition by the alcohol, with kinetic parameters obtained experimentally in liquid 2-methyl-2-butanol. These new values for the initial rates are equal to [25] :

$$
v_{i_{c a l c}}=\frac{V_{\max } a_{P} a_{M P}}{K_{M P}\left(1+\frac{a_{P}}{K_{I_{P}}}\right) a_{P}+K_{P} a_{M P}+a_{P} a_{M P}}
$$

Equation 2

The comparison between $v_{i_{c a l c}}$ and $v_{i_{\text {exp }}}$, and between $v_{i_{\text {pred }}}$ and $v_{i_{\text {exp }}}$, can be seen in Figure 1 , which presents experimental points and calculated and predicted lines for each different ap value. The fit of calculated lines to experimental points is much better (mean relative error of 
$1 \quad 10 \%$ ) than the fit of predicted lines (mean relative error of $56 \%$ ). Nevertheless it has to be

2 noticed that no actual measurement in organic media was used for this prediction, which

3 relies only on intrinsic parameters obtained in gas. The result of the prediction is a systematic

4 overestimation of initial rates. This must arise from more complex effects of solvent molecules on CALB catalyzed reaction, which seem to be more pronounced for strongly inhibiting solvents, like 2-methyl-2-butanol (see following part).

\subsection{Prediction of initial reaction rates, for CALB-catalyzed alcoholysis, in different liquid solvents, by using intrinsic parameters obtained in the solid/gas reactor}

In this last part, we tried to extend the use of intrinsic kinetic parameters and solvent intrinsic effect data, obtained in solid/gas reactor, for predicting reaction rates for CALBcatalyzed alcoholysis, in different liquid solvents and for different enzyme batches. It has to be pointed out that the following calculations are a very severe test of the efficiency of the prediction, as just solid/gas data and a single rate measurement in hexane are used to make predictions for other solvents and other enzyme batches.

By using the same approach as for 2-methyl-2-butanol, it was considered that the main origin of intrinsic solvent effect on enzyme catalysis arose from its ability to interact with the active site of the enzyme. A way of quantifying this interaction is to use the intrinsic inhibition constant of solvents (noted $K_{I_{\text {sovert }}}^{\circ}$ ), obtained as for 2-methyl-2-butanol in solid/gas reactor. These inhibition constants, determined for CALB-catalyzed alcoholysis of methyl propanoate and propanol, are given in Table 2, for water, 2-pentanone, 3-pentanone, 3hydroxy-3-methylpentane and hexane, in addition to 2-methyl-2-butanol. When the polarity of solvent, as estimated by $\operatorname{LogP}$, increases, $K_{I_{\text {solem }}}^{\circ}$ values decrease, which means that the inhibitory character of the solvent increases. The ability of polar compounds to interact with CALB active site has already been demonstrated $[12,16,26]$ and related to the polar nature of 
1 the active site region around Ser105 [27]. 2-methyl-2-butanol constitutes an exception to this

2 rule, as this tertiary alcohol causes more inhibition the the ketones 2-and 3-pentanone, while

3 being less polar.

4 For predicting initial reaction rate for our model reaction in the different solvents studied, the

5 equation 1 was used with the concerned solvent instead of 2-methyl-2-butanol.

6 For a new batch of CALB, the first step is to determine the intrinsic kinetic parameter $V_{\max }{ }^{\circ}$

7 According to equation $1, V_{\max }{ }^{\circ}$ can be obtained by experimentally measuring initial rate in

8 liquid medium with hexane as solvent, at known $a_{M P}$ and $a_{P}$. The term $\frac{a_{\text {solvent }}}{K_{\text {solvent }}^{\circ}}$ is neglected,

9 as hexane presents no inhibition of CALB-catalyzed alcoholysis.

$V_{\max }^{\circ}=\frac{v_{i}\left(K_{M P}^{\circ}\left(1+\frac{a_{P}}{K_{I_{P}}^{\circ}}\right) a_{P}+K_{P}^{\circ} a_{M P}+a_{P} a_{M P}\right)}{a_{P} a_{M P}}$

11 For $a_{M P}=0.5$ and $a_{P}=0.1, v_{i_{\mathrm{exp}}}=0.069 \mu \mathrm{mol} \cdot \mathrm{min}^{-1}$ per mg of immobilized CALB, and

$12 V_{\max }^{\circ}=0.125 \mu \mathrm{mol} \cdot \mathrm{min}^{-1} \mathrm{mg}^{-1}$.

13 For predicting initial rates for this enzyme batch, in a particular solvent and for chosen $a_{M P}$ 14 and $a_{P}$, the corresponding thermodynamic activity of the solvent has to be estimated by UNIFAC and $v_{i_{\text {pred }}}$ is obtained by using Equation 3.

16 Table 3 shows an example of initial rate predictions for the six solvents studied, for $a_{M P}=0.5$

17 and $a_{P}=0.1$, with the comparison with $v_{i_{\text {exp }}}$. The prediction method allowed to correctly order solvents with regard to CALB activity and gave good approximations of initial rate for 19 weakly inhibiting solvents. $v_{i_{\text {exp }}}$ tend to deviate from $v_{i_{\text {pred }}}$ for solvents showing a marked 20 inhibitor character. 


\section{Conclusion}

The influence of solvents on enzymatic reactions is complex and the use of solid/gas

4 reactor gives the opportunity to exclude one of the complicated effect induced by solvents,

5 that is substrate solubility issues. Once this effect is ruled out, the data shown in the present

6 study suggest that the influence of organic solvents on lipase-catalyzed alcoholysis could be

7 explained by the competitive inhibitory character of solvent molecules towards the first

8 substrate ester. This simplified scenario allowed to predict correctly initial reaction rates for

9 weakly inhibiting solvents, but gave rougher estimations for solvents with a more pronounced

10 inhibitory effect. More complex models have therefore to be constructed to fully understand

11 solvent effects. 


\section{Figure legend :}

3 Figure 1 : Effect of methyl propanoate thermodynamic activity on alcoholysis activity, at

4 different thermodynamic activity of propanol. Crosses correspond to experimental results

5 obtained in liquid 2-methyl-2-butanol at $70^{\circ} \mathrm{C}$, in $4 \mathrm{ml}$ of a solution containing methyl

6 propanoate, propanol and 2-methyl-2-butanol at specified thermodynamic activities, in

7 presence of $50 \mathrm{mg}$ adsorbed CALB onto Chromosorb AW DMCS. Full lines represent

8 calculated activities using the classical rate equation for the Ping Pong Bi Bi mechanism with

9 dead-end inhibition by the alcohol, with kinetic parameters obtained experimentally in liquid

10 2-methyl-2-butanol. Dashed lines represent predicted activities using the classical rate

11 equation for the Ping Pong Bi Bi mechanism with dead-end inhibition by the alcohol, with

12 intrinsic kinetic parameters obtained experimentally in the solid/gas reactor, and considering

13 that both 2-methyl-2-butanol and propanol were both acting as exclusive competitive

14 inhibitors of methyl propanoate. 
2 Table 1 : Dissociation constants of propanol enzyme, methyl propanoate enzyme and acyl-

3 enzyme propanol complexes respectively in the solid/gas reactor in absence of 2-methyl-2-

4 propanol (intrinsic parameters $K_{I_{P}}^{\circ}, K_{M P}^{\circ}$ and $K_{P}^{\circ}$ ) or in presence of a $2 \mathrm{M} 2 \mathrm{~B}$ equal to 0.3

$5 \quad\left(K_{I_{P}}^{0_{2} M 2 B}, K_{M P}^{0_{2 M 2 B}}\right.$ and $\left.K_{P}^{\circ_{2} M 2 B}\right)$ and in liquid 2-methyl-2-propanol $\left(K_{I_{P}}, K_{M P}\right.$ and $\left.K_{P}\right)$.

\begin{tabular}{|c|c|c|c|c|c|}
\hline & $a_{2 M 2 B}{ }^{\mathrm{a}}$ & $K_{I_{P}}^{\circ}$ & $K_{M P}^{\circ}$ & $K_{P}^{\circ}$ & $V_{\max }^{\circ}\left(\mu \mathrm{mol} \min ^{-1} \mathrm{mg}^{-1}\right)$ \\
\hline \multirow[t]{2}{*}{ Solid/gas reactor ${ }^{b}$} & 0 & 0.019 & 0.047 & 0.022 & $0.4^{\mathrm{e}}$ \\
\hline & & $K_{I_{P}}^{\circ 2 M 2 B}$ & $K_{M P}^{\circ 2 M 2 B}$ & $K_{P}^{\circ 2 M 2 B}$ & $V_{\max }^{\circ 2 M 2 B}\left(\mu \mathrm{mol} \mathrm{min}{ }^{-1} \mathrm{mg}^{-1}\right)$ \\
\hline \multirow[t]{2}{*}{ Solid/gas reactor $^{c}$} & 0.3 & 0.045 & 0.106 & 0.023 & $0.39^{f}$ \\
\hline & & $K_{I_{P}}$ & $K_{M P}$ & $K_{P}$ & $V_{\max }\left(\mu \mathrm{mol} \mathrm{min}^{-1} \mathrm{mg}^{-1}\right)$ \\
\hline Liquid medium ${ }^{b}$ & 0.33 to $0.96^{d}$ & 0.160 & 0.651 & 0.020 & $0.4^{\mathrm{e}}$ \\
\hline
\end{tabular}

6

$7 \quad{ }^{a}$ thermodynamic activity of 2-methyl-2-propanol

$8{ }^{\mathrm{b}}$ results from Bousquet-Dubouch et al. (2001) [16]

$9 \quad{ }^{c}$ results from Létisse et al. (2003) [12]

$10{ }^{\mathrm{d}}$ since all concentrations in liquid medium are linked together, data were obtained under the

11 following range of experimental conditions: $\mathrm{a}_{2 \mathrm{M} 2 \mathrm{~B}}=0.33\left(\mathrm{a}_{\mathrm{P}}=0.5\right.$ and $\left.\mathrm{a}_{\mathrm{MP}}=0.3\right)$, and $\mathrm{a}_{2 \mathrm{M} 2 \mathrm{~B}}$

$12=0.96\left(\mathrm{a}_{\mathrm{P}}=0.01\right.$ and $\left.\mathrm{a}_{\mathrm{MP}}=0.05\right)$

$13{ }^{\mathrm{e}} V_{\max }^{\circ}$ and $V_{\max }^{\mathrm{o} M 2 B}$ values were obtained from [16] after correction.

14 f unpublished result 
Table 2 : Intrinsic inhibition constants of solvents determined in a solid/gas reactor for

4 CALB-catalyzed alcoholysis of methyl propanoate and propanol.

5

6

7

8

9

\begin{tabular}{|l|c|c|}
\hline Solvent & Intrinsic inhibition constant & LogP \\
\hline water $^{\mathrm{a}}$ & 0.128 & $/$ \\
\hline 2-methyl-2-butanol & 0.22 & 1.22 \\
\hline 3-pentanone $^{\mathrm{c}}$ & 0.24 & 0.75 \\
\hline 2-pentanone $^{\mathrm{c}}$ & 0.29 & 0.75 \\
\hline 3-hydroxy-3-methylpentane $^{\mathrm{c}}$ & 7.03 & 1.71 \\
\hline Hexane $^{\mathrm{b}}$ & No inhibition & 3.29 \\
\hline
\end{tabular}

${ }^{\mathrm{a}}$ results from Bousquet-Dubouch et al. (2001) [16]

${ }^{\mathrm{b}}$ results from Létisse et al. (2003) [12]

${ }^{c}$ results from Graber et al. (2007) [15] 
2 Table 3 : Prediction of initial rates in water, 2-methyl-2-butanol, 3-pentanone, 2-pentanone,

3 3-hydroxy-3-methylpentane and hexane and comparison with experimentally measured initial

4 rates, for batch B of immobilized CALB with $a_{M P}=0.5$ and $a_{P}=0.1$, at $70^{\circ} \mathrm{C}$. The reaction

5 studied is alcoholysis of methyl propanoate and propanol.

\begin{tabular}{|c|c|c|c|}
\hline Solvent & $\begin{array}{l}\text { Thermodynamic } \\
\text { activity of solvent } \\
\text { for } \mathrm{a}_{\mathrm{MP}}=0.5, \mathrm{a}_{\mathrm{P}}=0.1 \text {, } \\
\text { estimated by } \\
\text { UNIFAC }\end{array}$ & $\begin{array}{l}v_{i_{\text {pred }}}(\mu \mathrm{mol} / \mathrm{min} . \mathrm{mg}) \\
\text { with } V_{\max }{ }^{\circ}=0.125 \mu \mathrm{mol} \cdot \mathrm{min}^{-1} \mathrm{mg}^{-1} \text {, } \\
\text { obtained in hexane }\end{array}$ & $v_{i_{\exp }}\left(\mu \mathrm{mol} \cdot \mathrm{min}^{-1} \mathrm{mg}^{-1}\right)$ \\
\hline $\begin{array}{l}\text { 2-methyl-2- } \\
\text { butanol }\end{array}$ & 0.607 & 0.06 & 0.031 \\
\hline 3-pentanone & 0.49 & 0.0625 & 0.047 \\
\hline 2-pentanone & 0.48 & 0.063 & 0.061 \\
\hline $\begin{array}{l}\text { 3-hydroxy-3- } \\
\text { methylpentane }\end{array}$ & 0.535 & 0.068 & 0.067 \\
\hline hexane & 0.657 & 0.069 & 0.069 \\
\hline
\end{tabular}




\section{References}

3

4

5

6

7

8

9

10

11

12

13

14

15

16

17

18

19

20

21

22

23

24

26

27

3
4 6

25 [9] J. Kim, D. S. Clark, J. S. Dordick, Biotechnol. Bioeng. 67 (2000) 112-116.

[1] G. Bell, P.J. Halling, B.D. Moore, J. Partridge and D.G. Rees, Trends Biotechnol. 13 (1995) 468-473.

[2] J.S. Dordick, in: H.W. Blanch, D.S. Clark (Eds.), Applied biocatalysis, Marcel Dekker, New York, 1991, pp.1-51.

[3] C. Laane, S. Boeren, K. Vos and C. Veeger, Biotechnol. Bioeng. 30 (1987) 81-87.

[4] P. J. Halling, Curr. Opin. Chem. Biol. 4 (2000) 74-80.

(1)

[5] K. Watanabe, T. Yoshida, S. Ueji, Bioorganic Chemistry 32 (2004) 504-515.

(1)

[6] A.E.M. Janssen, B.J. Sjursnes, A.V. Vakurov and P.J. Halling, Enzyme Microb. Technol. $24(1999) 463-470$

8

[7] J.B.A. Van Tol, R.M.M. Stevens, W.J. Veldhuizen, J.A. Jongejan and J.A. Duine, Biotechnol. Bioeng. 47 (1995) 71-81.

1

22

[8] M. Martinelle, K. Hult, Biochim. Biophys. Acta 1251 (1995) 191-197.

7 [10] L. F. Garcia-Alles, V. Gotor, Biotechnol. Bioeng. 59 (1998) 163-170. 
2 [11] S. Lamare and M.D. Legoy, Biotechnol. Bioeng. 45 (1995) 387-397.

3

4 [12] F. Létisse, S. Lamare, M.D. Legoy and M. Graber, Biochim. Biophys. Acta - Proteins \& $5 \quad$ Proteomics 1652 (2003) 27-34.

7 [13] S. Lamare, M.D. Legoy and M. Graber M., Green Chem. 6 (2004) 445-458.

8

9 [14] L. Olofsson, I. A. Nicholls, S. Wikman, Org. Biomol. Chem. 3 (2005) 750-755.

10

11 [15] M. Graber, R. Irague, E. Rosenfeld, S. Lamare, L ; Franson, K. Hult, Biochim. Biophys. 12 Acta 1774 (2007) 1052-1057.

13

14 [16] M.P. Bousquet-Dubouch, M. Graber, N. Sousa, S. Lamare and M.D. Legoy, Biochim. Biophys. Acta 1550 (2001) 90-99.

16

[17] R.P. Bell, J.E. Critchlow and P. M.I. Page, J. Chem. Soc., Perkin Trans. II (1974) 66-70.

18

19 [18] J.B.A.Van Tol, J.B. Odenthal, J.A. Jongejan and J.A. Duine, in: J. Tramper, M. H. 20 Vermuë,H. H. Beeftink and U. Von Stockar Eds. Biocatalysis in non-conventional media, 21 Elsevier, Amsterdam 1992, pp. 229-235.

23 [19] J. S. Dordick, Biotechnol. Prog. 8 (1992) 259-267.

24 
2 [21] A.E.M. Janssen, A.M. Vaidya and P.J. Halling, Enzyme Microb. Technol. 18 (1996) $3 \quad 340-346$.

4

[22] P. J. Halling, Enzyme Microb. Technol. 16 (1994) 178-206.

6

7 [23] H.K. Hansen, P. Rasmussen, A. Fredenslund, M. Schiller and J. Gmehling, Ind. Eng. 8 Chem. Res. 30 (1991) 2355-2358.

9

[24] J. L. Schmitke, L. J. Stern, A. M. Klibanov, Proc. Natl. Acad. Sci. U.S.A. 95 (1998) $11 \quad 12918-12923$.

12

[25] I. H. Segel, Enzyme kinetics - Behavior and analysis of rapid equilibrium and steadystate enzyme systems, John Wiley and Sons, New York, 1975.

[26] M. Graber, M. P. Bousquet-Dubouch, S. Lamare and M.D. Legoy, Biochim. Biophys. Acta 1648 (2003) 24-32.

18

[27] J. Uppenberg, N. Ohrner, M. Norin, K. Hult, G.J. Kleywegt, S. Patkart, V. Waagen, T. Anthonsen and T.A. Jones, Biochemistry 34 (1995) 16838-16851. 
$1 \quad$ Figure 1
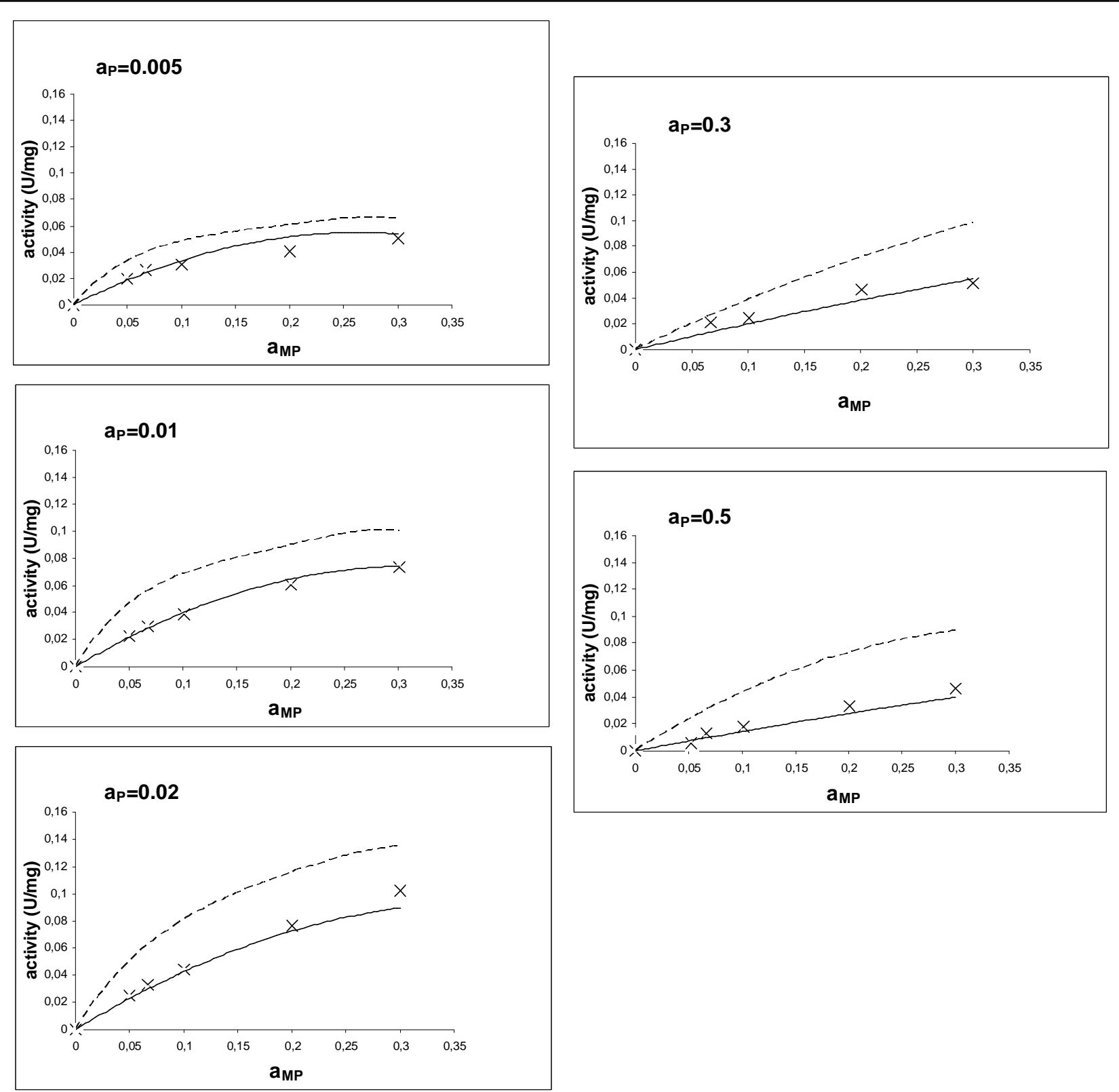